\title{
Lesión muscular en podología. Actualización en patofisiología y terapéutica
}

\section{Muscle injury in podiatry. Update on pathophysiology and therapeutic}

\author{
David Rodríguez SAnz ${ }^{1,2,3,6}$, Daniel López López ${ }^{2,5,7}$, \\ Patricia Palomo López ${ }^{2,4,6}$, Alfredo Soriano Medrano ${ }^{2,6}$, \\ Ángel Morales Ponce ${ }^{2,6}$ \\ 1. Diplomado en Fisioterapia. \\ 2. Diplomado en Podología. \\ 3. Profesor Universidad Europea de Madrid. \\ 4. Profesor Universidad Extremadura. \\ 5. Profesor Universidad A Coruña. \\ 6. Doctor por la Universidad Rey Juan Carlos. \\ 7. Doctor por la Universidad A Coruña. \\ davidrodriguezsanz@hotmail.com; daniellopez@udc.es; patibiom@unex.es; soriano.alfredo@gmail.com; \\ clinicatorrijos@gmail.com
}

\author{
Correspondencia: \\ David Rodríguez Sanz \\ C/Embajadores $1965 \mathrm{E}$ \\ E-Madrid 28045 \\ Correo electrónico: davidrodriguezsanz@hotmail.com
}

Fecha de recepción: 8 de julio de 2013

Fecha de aceptación: 22 de diciembre de 2013

Los autores declaran no tener ningún tipo de interés económico o comercial.

\section{RESUMEN}

El tejido muscular es de elevado interés cientifico en podología debido a su influencia en el mantenimiento de la postura y la deambulación, siendo necesario su perfecto funcionamiento para poder desarrollar dichas actividades con la eficacia y eficiencia esperadas. Además se encuentra fuertemente asociada al desarrollo de actividades deportivas. Son muy frecuentes las lesiones en su estructura muscular que pueden alterar dichas actividades funcionales. A continuación realizaremos un trabajo de actualización en las lesiones musculares y profundizaremos más en el DOMS (Delayed Onset Muscle Soreness) de elevado interés biológico por su información en la reparación biológica de los tejidos dañados.

Palabras clave: regeneración; biología; músculo; lesiones.

\section{ABSTRACT}

Muscle tissue is of high scientific interest in podiatry due to its influence on the maintenance of posture and gait, perfect operation being necessary to develop such activities expected effectiveness and efficiency. Besides is strongly associated with the development of sports. They are very frecuents lesions in muscular structures that can alter these functional activities. Then do a major upgrade in muscle strains and delve more into the DOMS (Delayed Onset Muscle Soreness) of high biological interest for its information on the biological repair of damaged tissue.

Keywords: regeneración; biology; muscle; injuries.

Sumario: 1. Lesión muscular. Introducción. 2. Práctica clínica en tratamiento de lesiones musculares en podología. 3. Conclusión. Bibliografía.

Referencia normalizada: Rodríguez Sanz, D., López López, D., Palomo López, P., Soriano Medrano, A., Morales Ponce, Á.. Lesión muscular en podología. Actualización en patofisiología y terapéutica. Rev. Int. Cienc. Podol. 2015; 9(2): 99-105. 


\section{LESIÓNMUSCULAR.INTRODUCCIÓN}

El músculo es un tejido que constituye la anatomía humana y que frecuentemente se ve lesionado, con una demografía de lesiones que puede llegar al $55 \%$ del total de lesiones producidas en todo el cuerpo ${ }^{1}$. Existen numerosos autores de carácter internacional como Jarvinen con una elevada especialización en este tipo de patología, con numerosas publicaciones y de solido interés cientifico, el cual es una referencia directa en la realización de esta obra ${ }^{1}$. Nos encontramos diferentes clasificaciones de las lesiones musculares en función del tipo de tejido, del mecanismo de acción lesiva desencadenante o de la gravedad del daño ocurrido en los tejidos. Por ejemplo, podemos encontrar desgarros musculares, donde observamos lesión en las fibras musculares, en lámina basal y su envoltura u otras lesiones como la rabdomiolisis donde la fibra muscular se lesiona pero lámina basal y envoltura se mantienen íntegras ${ }^{1,2,3,4}$. Si bien es cierto, que la mayoría de las lesiones musculares asociadas a la actividad deportiva, se provocan por lesiones que presentan mecanismos de contusión asociados ${ }^{1}$. Así pues, haciendo referencia al origen del mecanismo lesivo, podríamos diferenciar dos grandes grupos de lesiones musculares, un grupo de carácter intrínseco, asociado a la actividad muscular y otro grupo extrínseco asociado a elementos externos. Dentro de las lesiones de componente intrínseco y gracias a los estudios histológicos de última generación, encontramos el dolor muscular de aparición tardía, Delayed Onset Muscle Soreness (DOMS) de especial interés por su asociación a los factores de entrenamiento en poblaciones que realizan actividad física. Si bien es cierto que el podólogo no encontrara estos cuadros en su fase más aguda, debido al auge de la actividad deportiva en la población, como por ejemplo el running, es de interés que conozcamos los pormenores de la patofisiologia de la lesión así como las opciones terapéuticas de la podología física.

\section{CLASIFICACIÓN CLÍNICA DE LAS DIS- TENSIONES MUSCULARES. NUEVAS PERSPECTIVA HISTOLÓGICA. DOMS}

La evolución y progresión clínica de la lesión muscular dependerá, por lo tanto, de varios fac- tores, por un lado asociados al propio evento lesivo y por otro lado asociados a las condiciones propias del sujeto, así pues encontraremos entre otros factores, la cantidad de tejido dañado, la naturaleza de la destrucción del músculo, el medio ambiente en el que se desarrolla el proceso de regeneración del tejido.

El DOMS, puede considerarse como la forma más leve de lesión por esfuerzo. Basándonos en las repercusiones clínicas y funcionales, las lesiones musculares se pueden clasificar como leves, moderadas o graves ${ }^{5,6}$. Atendiendo a una cuantificación tisular encontraremos, lesión leve (también denominada de primer grado) implica ruptura de pocas fibras musculares, se asocia a pequeñas molestias y discreta inflamación, acompañado de ninguna o de una pérdida de fuerza y de limitación funcional mínima. Una lesión moderada (también denominada de segundo grado) supone un daño estructural mayor del músculo con limitación funcional asociada al estiramiento y a la contracción del segmento lesionado. Una lesión grave (también denominada de tercer grado) se produce cuando un desgarro se extiende en toda la sección transversal del músculo (una consecuencia muy rara si únicamente actúa una fuerza intrínseca excesiva) lo que implica una pérdida casi completa de la función muscular.

El DOMS, se trata por tanto, de una lesión muscular leve, la cual se encuentra asociada al ejercicio físico, de manera general aparece como consecuencia de un ejercicio demasiado intenso en un músculo no entrenado, es una lesión que permite tolerancia a la actividad física y provoca dolor muscular entre las 24 y las 72 horas después del mismo. Este fenómeno se produce especialmente si el ejercicio realizado incluye actividad de componente muscular excéntrico. Por lo que puede ser habitual encontrarlo en pacientes que han realizado actividad de carrera y acuden a consulta en los días posteriores.

La clínica habitual del DOMS cursa de forma general con rigidez, dolor y molestias a la palpación directa durante las primeras 48 horas, con un pico de dolor entre las 48 y las 72 horas, y que en general desaparece sin necesidad de tratamiento a partir del 5 día. El dolor aumenta por el estiramiento muscular 
pasivo encontrándose asociada una disminución de la fuerza muscular. Biológicamente, se encuentra un aumento de la creatinina sérica $(\mathrm{CK})$, que puede ver aumentado su valor basal 20 veces. Este valor aumentado alcanza su pico entre los días 3 al 6 y regresa a sus valores normales tras una semana del ejercicio excéntrico. En estudios histológicos, encontramos que aparece reacción inflamatoria en animales, y en menor grado en humanos ${ }^{7}$. El dolor en el DOMS está mediado por nociceptores tipo III y IV, los cuales son estimulados por factores de componente inflamatorio (tales como la bradiquinina, prostaglandinas y serotonina). Por tanto encontramos de manera frecuente el uso de fármacos antiinflamatorios no esteroideos (AINEs) para reducir el dolor, pero respecto a la inflamación al ser poca, no necesita de reducción por vía farmacológica. Sobre la patofisiologia del DOMS, este se desarrolla tras un trabajo excéntrico excesivo para el rendimiento normalizado de dicho musculo. Respecto a las lesiones musculares de mayor profundidad en experimentación animal, se ha demostrado que el estiramiento estimula a las células satélite inactivas $\mathrm{y}$ favorecer la división de las mismas ${ }^{8}$ lo cual conlleva la regeneración de la zona lesionada. Por tanto encontramos como lo estímulos mecánicos directos son capaces de favorecer la diferenciación del tejido y completar la dirección del mismo como mecanismos básicos para su correcta diferenciación. Este efecto biológico se define como mecanotransduccion y explica el fenómeno fisiológico de la diferenciación celular en función de los estímulos mecánicos a los que se ve sometido el segmento.

En el Doms se encuentra un bajo nivel de destrucción tisular y se ha observado que la descendencia de las células satélite activadas no maduraba en mioblastos, ni expresaba proteínas específicas musculares ni se fusionaba con la miofibra parental debido a que no había necesidad de regeneración estructural muscular ${ }^{8}$. A pesar de que el DOMS se asocia con el aumento de CK y que este es un indicador de daño sarcolemico el cual que induce la pérdida de proteínas sarcoplásmicas, se ha demostrado que el DOMS no existe lesión de miofibrillas ${ }^{8,9}$.

\section{PRÁCTICA CLÍNICA EN TRATA- MIENTO DE LESIONES MUSCULARES EN PODOLOGÍA}

Diferenciaremos dos procesos, el primero en el que se incluirá el tratamiento precoz en la fase más inicial de la enfermedad, que buscara minimizar el daño y las complicaciones clínicas y a continuación un proceso posterior donde el podólogo contara con las herramientas de la podología física para la recuperación optima de la lesión muscular.

\subsection{Tratamiento precoz de la lesión \\ 2.1.1. Método Rice}

El tratamiento inmediato y precoz, del músculo esquelético lesionado (y en general de cualquier lesión de los tejidos blandos) se conoce como el principio "RICE": Reposo, Hielo Compresión y Elevación. La justificación clínica de reside de manera directa en la pretensión de la reducción del sangrado en la zona de tejido dañado para evitar complicaciones posteriores asociadas. Es necesario destacar que no existe ningún ensayo clínico aleatorizado (ECA) que valide la eficacia del principio "RICE" en el tratamiento de lesiones de tejidos blandos ${ }^{10}$. Sin embargo, si existen pruebas científicas sobre la adecuación de sus distintos componentes de manera individual y por separado, derivándose en esta dirección, la evidencia en gran parte de estudios experimentales. La prueba más sólida para la recomendación clínica del reposo viene dada a partir de estudios sobre los efectos de la inmovilización en la curación de los músculos ${ }^{1,2}$. El reposo, tras el acontecimiento lesivo, se puede evitar la posterior retracción de los extremos de la rotura, lo que reduce el tamaño del hematoma y, consecuentemente, el tamaño del tejido cicatricial ${ }^{1,2}$. Respecto al uso de frío terapéutico en la lesión, se observa que el uso precoz de crioterapia está asociada con reducción significativa del hematoma en los extremos de la rotura miofibrilar, con menos inflamación y necrosis tisular acelerando la regeneración temprana ${ }^{11,12,13}$. Respecto a la compresión, aunque provoca que disminuya el flujo de sangre por vía intramuscular en la zona lesionada ${ }^{14}$, es discutible y no se conoce con solida certeza si la compresión aplicada 
tras de la lesión acelera la cicatrización del músculo esquelético lesionado ${ }^{14}$.

Sin embargo, las guías clínicas, recomiendan y mantienen que el efecto sumado de hielo (crioterapia) y compresión se apliquen en sesiones de 15 a 20 minutos de duración, repetidos a intervalos de 30 a 60 minutos por lo menos durante un periodo mínimo de 6 horas para obtener efecto sustancial sobre la limitación de la hemorragia y necrosis de los tejidos en el sitio de la lesión ${ }^{13}$.

Por último, en relación con el último componente del principio RICE, la elevación, la justificación de su uso se basa en los principios básicos fisiológicos de mecánica de fluidos; la elevación de la extremidad lesionada por encima del nivel del corazón provoca una disminución de la presión hidrostática y, por tanto, reduciendo de manera directa la posibilidad de la acumulación de líquido intersticial.

\subsection{Tratamientoposteriordelalesión muscular}

Si la evolución se muestra favorable, el tratamiento de componente más activo de la extremidad lesionada se comenzara de forma gradual. Solo existe un solo estudio prospectivo y aleatorizado en la literatura comparando diferentes regímenes de tratamiento para las lesiones musculo esqueléticas ${ }^{15}$. El estudio sobre lesiones en musculatura isquiotibial, de interés en podología por su relación directa con bipedestación y locomoción humana, ofrece un protocolo que consiste en ejercicios de flexibilidad progresiva añadiendo estabilización en cintura pélvica y lumbar, el cual obtiene un resultado significativamente mejor (reducción de recidivas y retorno más rápido a la actividad deportiva) que un protocolo centrado en estiramiento $\mathrm{y}$ fortalecimiento de la musculatura isquiotibial lesionada ${ }^{15}$. Esto nos lleva a pensar que es de interés las relaciones sinérgicas biomecánicas que encontramos en la zona dañada con la musculatura y articulaciones vecinas.

\subsubsection{Calentamiento (Warm Up)}

Como recordatorio inicial, es de particular importancia tener en cuenta que las actividades de recuperación física podológica en lesiones musculares deben comenzar siempre con un adecuado calentamiento del músculo lesionado ${ }^{16,17}$, el adecuado calentamiento se ha demos- trado de elevada utilidad en la reducción de la viscosidad de muscular, además de generar una relajación de componente neurológico. Además, debido a los principios estructurales de colágeno y elastina, conocemos que los músculos calentados y estimulados pueden absorber más energía que los músculos sin estimulación lo que nos lleva a un mejor soporte de la carga ${ }^{17}$. Podremos usar medios físicos como la carga controlada o el uso de lámparas de infrarrojos.

\subsubsection{Estiramiento (Stretching)}

Es conocido que el estiramiento aumenta de los músculos ${ }^{17,18}$. Uno de los fines que promueve el estiramiento es favorecer la remodelación funcional, dotando a un tejido que está en fase de crecimiento y de diferenciación tisular, de aquellos estímulos mecánicos adecuados para que dicha diferenciación desemboque en un resultado óptimamente funcional, para que la cicatriz que se encuentra en una fase todavía plástica, pero en la que ya tiene la fuerza suficiente necesaria para evitar una retracción funcionalmente incapacitante de los bordes del músculo, esto se conoce con el nombre de mecanotrasduccion. El estiramiento sin dolor de la cicatriz madura puede lograrse mediante estiramiento gradual, comenzando con series de 10 a 15 segundos cada vez y luego aumentar a un periodo de 1 minuto. El estiramiento también debe incluir estiramientos repetidos del mismo músculo debido a que la elongación muscular repetida ha demostrado que disminuye la contrarresistencia del músculo al estiramiento ${ }^{16}$.

\subsubsection{Farmacoterapia}

Existen pocos estudios controlados aleatorizados sobre el uso de medicamentos antiinflamatorios no esteroideos (AINEs) así como del uso de glucocorticoides en el tratamiento de lesiones musculares en los seres humanos. Existe un estudio sobre el uso de AINEs en el tratamiento, lesiones de las miofibrillas. En este tipo menos grave de lesión muscular, un uso a corto plazo de AINEs oferta una mejora transitoria en la recuperación de la lesión muscular inducida por el ejercicio ${ }^{19}$. A pesar de la falta de evidencia específica, los efectos de los AINEs han sido bien documentados experimentalmente ${ }^{20,21,22}$. El uso a corto plazo de AINEs en la primera fase de curación ha mostrado una disminución en 
la reacción de las células inflamatorias ${ }^{20,22} \sin$ efectos adversos en el proceso de curación, en la resistencia a la tracción o en la capacidad del músculo lesionado para contraerse ${ }^{20}$. Además, los AINEs no retardan la regeneración de miofibras $^{22}$. Mientras que el uso temprano a corto plazo de los AINEs se puede considerar un tratamiento relativamente bien justificado ${ }^{20,21,22}$ la situación parece ser totalmente contraria respecto a los glucocorticoides ${ }^{20,23}$. Tras el uso de estos medicamentos para el tratamiento de lesiones musculares se ha observado retraso en la eliminación del hematoma y del tejido necrótico, un retraso del proceso de regeneración del músculo y en ultima estancia la reducción de la fuerza biomecánica del músculo lesionado ${ }^{20,23}$.

\subsubsection{Electroterapia terapéutica. Corrientes antialgicas y ultrasonido terapéutico}

El ultrasonido terapéutico (US) se recomienda y usa frecuentemente en el tratamiento de lesiones musculares en numerosas guías clínicas, aunque los datos científicos obtenidos a partir de estudios en animales no muestran eficacia sustancial científicamente soportada ni evidencia clínica para la recomendación directa de este perfil clínico, si bien es cierto que los estudios no poseen calidad metodológica suficiente, sobre modo de aplicación, dosificación y patrones de desarrollo ${ }^{24,25,26} \mathrm{Sin}$ embargo, a pesar de la aparente promoción de la regeneración de tejido basado en los efectos mecánicos y térmicos del US, durante la fase de proliferación de la mi regeneración ${ }^{24}$, el US terapéutico no parece tener efecto positivo en el resultado final de la curación muscular ${ }^{24,25,26}$ por lo que se precisan de estudios solidos que aporten un mayor conocimiento del mismo.

El control del dolor está relacionado con la disminución de la inflamación, para eliminar estas sensaciones dolorosas podremos usar electroterapia con corrientes tipo Tens, o láser y magnetoterapia con este fin. En función del tipo de fibra dañada estarán indicadas un tipo de tratamiento electroterápico u otro. En general cuando se lesionan fibras rápidas serán rápidos los procedimientos y cuando son fibras lentas las dañadas serán procedimientos lentos, así encontraremos las siguientes frecuencias de electro estimulación $16 \mathrm{~Hz}$ cuando sean fibras lentas, $33 \mathrm{~Hz}$ en intermedias, $66 \mathrm{~Hz}$ en fibras rápidas y $80 \mathrm{~Hz}$ cuando se traten de fibras musculares ultrarrápidas.

\subsubsection{Masaje transverso profundo (Cyriax)}

Denominada maniobra de Cyriax, también colabora en la orientación de las fibras, favorece la formación de una cicatriz más elástica y disminuye las adherencias fibrosas además de favorecer el desplazamiento sobre planos adyacentes y todos los beneficios nombrados anteriormente, es una técnica de ayuda que permitirá al podólogo favorecer la adecuada remodelación del tejido y que no existan limitaciones accesorias.

\subsubsection{Recuperación Articular}

Será de interés para la óptima evolución clínica de nuestros pacientes y antes de completar los tratamientos de fortalecimiento en los músculos afectados, ayudar a la vuelta a la normalidad de las articulaciones sobre las que actuaremos. Es de interés recordar que estas articulaciones en ocasiones han permanecido inmovilizadas mediante unas férulas durante un periodo de tiempo en los casos más severos de las roturas musculares con procesos quirúrgicos asociados, y sin llegar a completar sus arcos fisiológicos de movimiento.

Es posible que aparezcan adherencias articulares, periarticulares, capsuloligamentosas e incluso de las unidades miotendinosas que no estaban implicadas en un primer momento en la lesión.

Es interesante comenzar con un agente que genere cierta hiperemia en las estructuras que vamos a tratar. Una vez realizado este calentamiento articular pasivo pasaremos a usar métodos cinesiterapicos para completar el arco normal de la articulación, en función del estadio en que la encontremos, precisáremos de un tratamiento con cinesiterapia forzada o pasiva o la modalidad que el clínico estime oportuna.

\subsection{Vuelta a la actividad previa}

La decisión más crucial en el tratamiento de pacientes con lesiones de tejido muscular esquelético es la decisión clínica del retorno a la actividad previa sin provocar una rotura recurrente, recaídas o complicaciones posteriores. En la práctica clínica la decisión debe basarse en toda la información sobre el evento lesivo, 
así como el lugar, la calidad base del tejido y la gravedad de la lesión. Hasta hace poco no había medidas fiables para predecir el tiempo de inactividad del atleta tras una lesión muscular grave. Sin embargo, ${ }^{29}$ mostraron que cuatro medidas simples directas funcionales en el examen clínico, realizadas de 3 a 5 días tras la lesión, predicen un proceso de recuperación superior a 4 semanas.

1. Alto nivel de dolor que persiste

2. Sensibilidad a la palpación

3. Dolor posterior a 72 horas en las actividades cotidianas

4. Dolor en posición de estiramiento base.

La decisión sobre el momento adecuado del retorno a la actividad previa específica puede estar basado en dos medidas simples de carácter clínico: la capacidad de estirar el músculo lesionado tanto como el músculo contralateral sano y la contracción sin dolor del músculo lesionado en los movimientos básicos. Cuando el tejido se encuentra en este escenario se puede comenzar con el retorno progresivo a la actividad previa ${ }^{30}$.

\section{CONCLUSIÓN}

Existen pocos estudios clínicos desarrollados con calidad metodológica suficiente, sobre el tratamiento de las lesiones musculares y, por lo tanto, los principios actuales de tratamiento se basan en su mayoría en estudios experimen- tales o en la evidencia empírica exclusivamente. Clínicamente, los primeros auxilios en las lesiones musculares siguen el principio RICE (reposo, hielo, compresión y elevación), el principio común para el tratamiento de cualquier trauma de tejidos blandos con evidencia parcial del mismo. Durante los primeros días después de la lesión, un corto período de inmovilización, entendida como inmovilización funcional, preventiva para evitar daños posteriores, y no tratándose en ningún caso de una inmovilización total del tejido, acelera la formación de tejido de granulación en el lugar de la lesión, pero se debe tener en cuenta que la duración de la reducción de la actividad (inmovilización) debe durar exclusivamente hasta que la cicatriz tenga suficiente fuerza para soportar las fuerzas de tracción inducidas por la contracción muscular sin volver a romperse, pues son precisamente los estímulos mecánicos adecuados al tejido proliferativo el que actuara sobre la lesión regenerando la zona dañada, favoreciendo proliferación y diferenciación de tejido y logrando una sólida. El uso de medidas de podología física por parte del podólogo en aquellas lesiones que afecten a la musculatura del miembro inferior es de elevado interés, pues le permitirá ofertar un mayor abanico de posibilidades terapéuticas y lograr mejores resultados clínicos en una población que debido al auge de la actividad deportiva cada vez presenta mayor actividad deportiva y más lesiones asociadas a dicha actividad como son las lesiones musculares.

\section{BIBLIOGRAFÍA}

1. Järvinen T, Järvinen T, Kääriäinen M, Kalimo H, Järvinen M. Biology of muscle trauma. Am J Sports Med. 2005;33:745-766.

2. Kuang S, Kuroda K, Le Grand F, Rudnicki MA. Asymmetric self-renewal and commitment of satellite stem cells in muscle. Cell. 2007;129:999-1010.

3. Huard J, Li Y, Fu F. Muscle injuries and repair: current trends in research. Journal of Bone \& Joint Surgery. 2002;84-A:822-832.

4. Beiner JM, Jok1 P. Muscle contusion injury and myositis ossificans traumatica. Clin Orthop Rel Res .2002;403S:S110-S119.

5. Kalimo H, Rantanen J, Järvinen M. Muscle injuries in sports. Baillière's Clinical Ortho.1997;2:1-24.

6. Jackson DW, Feagin J. Quadriceps contusions in young athletes: relation of severity of injury to treatment and prognosis. J Bone \& Joint Surg. 1973; 55-A:95-105.

7. MacIntyre NJ, Bhandari M, Blimkie CJ, Adachi JD \& Webber CE. Effect of altered physical loading on bone and muscle in the forearm. Can J physiol pharmacol. 2001;79:1015-1022.

8. Äärimaa V, Rantanen J, Heikkilä J, Helttuala I, Orava S. Rupture of the pectoralis major muscle. Am J Sports Med.2004;32:1256-1262. 
9. Yu JG, Thornell LE. Desmin and actin alterations in human muscles affected by delayed onset muscle soreness: a high resolution immunocytochemical study. Histochem Cell Biol. 2002;118:171-9.

10. Bleakley C, McDonough S, MacAuley D. The use of ice in the treatment of acute soft tissue injury: A systematic review of randomized controlled trials. Am J Sports Med. 2004;34:251-261.

11. Hurme T, Rantanen J \& Kalimo H.Effects of early cryotherapy in experimental skeletal muscle injury. Scand J Med \& Sci Sports.1993;3:46-51.

12. Deal DN, Tipton J, Rosencrance E, Curl WW, Smith TL. Ice reduces edema. A study of microvascular permeability in rats. J Bone \& Joint Surg . 2002;84-A:1573-1578.

13. Schaser KD, Disch AC, Stover JF, Lauffer A, Bail HJ, Mittlmeier T. Prolonged superficial local cryotherapy attenuates microcirculatory impairment, regional inflammation, and muscle necrosis following closed soft tissue injury in rats. Am J Sports Med. 2007;35:93-102.

15. Sherry MA, Best TM. A comparison of 2 rehabilitation programs in the treatment of acute hamstring strains. J Orthop Sports Phys Ther. 2004; 34;116-125.

16. Petersen J, Hölmich P. Evidence based prevention of hamstring injuries in sports. Br J Sports Med. 2005;39:319-323.

17. Safran MR, Garrett WE Jr, Seaber AV, Glisson RR \& Ribbeck BM.The role of warm-up in muscular injury prevention. Am J Sports Med. 1988;16:123-129.

18. Noonan TJ, Best TM, Seaber AV \& Garrett WE Jr. Thermal effects on skeletal muscle behavior. Am J Sports Med. 1993; 21:517-522.

19. O'Grady M, Hackney AC, Schneider K, Bossen E, Steinberg K, Douglas JM Jr, Murray WJ, Watkins WD. Diclofenac sodium (Voltaren) reduced exercise-induced injury in human skeletal muscle. Med Sci Sports \& Exerc. 2002;32:1191-1196.

20. Järvinen M, Lehto M, Sorvari T. Effect of some anti-inflammatory agents on the healing of ruptured muscle. An experimental study in rats. J Sports Traumatol.1992;14:19-28.

21. Rahusen FT, Weinhold PS, Almekinders LC. Nonsteroidal anti-inflammatory drugs and acetaminophen in the treatment of an acute muscle injury. Am J Sports Med. 2001;32:1856-1859.

22. Thorsson O, Lilja B, Nilsson P, Westlin N. Immediate external compression in the management. Scand J Med \& Sci Sports. 1998;7:182-190.

23. Beiner JM, Jokl P, Cholewicki J. The effects of anabolic steroids and corticosteroids on healing of muscle contusion injury. Am J Sports Med. 1999; 27:2-9.

24. Rantanen J, Thorsson O, Wollmer P, Hurme T, Kalimo H. Effects of therapeutic ultrasound on the regeneration of skeletal muscle myofibers after experimental muscle injury. Am J Sports Med. 1999; 27:54-59.

25. Wilkin LD, Merrick MA, Kirby TE, Devor ST. Influence of therapeutic ultrasound on skeletal muscle regeneration following blunt contusion. Int J Sports Med. 2004; 25:73-77.

26. Markert CD, Merrick MA, Kirby TE, Devor ST. Nonthermal ultrasound and exercise in skeletal muscle regeneration. Arch Phys Med Rehabil. 2005; 86:1304-1310.

27. Saraux et al. Clinical Predictors of Time to Sports Resumption in Muscle Injuries, submitted of an acute muscle injury. Scand J Med \& Sci Sports. 2011; 7:182-190.

28. Kujala UM, Orava S \& Järvinen M. Hamstring injuries: Current trends in treatment and prevention. Sports Med.1997; 23:397-404. 